http://jmscr.igmpublication.org/home/ ISSN (e)-2347-176x ISSN (p) 2455-0450 crossref DOI: https://dx.doi.org/10.18535/jmscr/v8i6.15

\author{
Journal Of Medical Science And Clinical Research

\title{
Pure Neuritic Leprosy with Ichthyosis like features-A Case Report
}

\author{
Authors \\ Dr Pooja K ${ }^{1 *}$, Dr M.S. Srinivasan ${ }^{2}$ \\ ${ }^{1}$ Junior Resident, ${ }^{2}$ Head of the Department \\ Department of Dermatology, Venereology and Leprosy, Chettinad Hospital and Research Institute, \\ Kelambakkam, India \\ *Corresponding Author
}

Dr Pooja K

Junior Resident, Department of Dermatology, Venereology, and Leprosy, Chettinad Hospital and Research

Institute, Kelambakkam, India

\begin{abstract}
Introduction: Pure neuritic leprosy is characterized by sensory and motor changes in leprosy in the absence of acid fast lepra bacilli on histopathology.

Objectives: To understand the neurological complications of leprosy which can occur due to the lack of complete \& proper treatment \& the clinical and histological features of patient with pure neuritic leprosy.

Methods: Here we will discuss a case report of a 23 year old male patient who presented with complaints of decreased sensation, weakness and thinning of right hand gradually over a period of 10 years.

History of multibacillary leprosy before 10 years with irregular treatment.

On dermatological examination, there was generalized xerosis noted over the dorsum of right hand \& forearm with finely scaly, ichthyosis like skin changes.

On neurological examination, there was decreased sensation over the dorsum of right hand along with motor deficits. Thickening of ulnar nerve present on palpation.

Results: Patient was advised high resolution ultrasound examination of both upper limbs which revealed thickening of ulnar nerve present over both upper limbs.

Skin biopsy was done \& histopathology reveals perivascular inflammatory infiltrate in the dermal vessels, dermal fibrosis \& thickening.

Conclusion: Even though leprosy was previously eliminated many years ago in India, there has been a drastic increase recently in the reporting of incidence of new cases of leprosy and cases with residual deformities due to leprosy.

Hence, it is very important on the part of clinicians \& health care workers to strictly monitor the treatment \& follow up of the patient in order to prevent deformities.

Keywords: Leprosy, Acid fast bacilli, pure neuritic leprosy.

Key Messages: In order to prevent the serious complications that can occur due to leprosy, it is important to know the steps in the management of leprosy, which will ease the diagnosis \& facilitate quick intervention.
\end{abstract}




\section{Introduction}

Pure neuritic leprosy is characterized by the occurrence of sensorineural \& motor deficits in the patient without obvious skin findings of leprosy.

There has been reported prevalence of upto $8 \%$ cases of pure neuritic leprosy in India.

Since there is absence of skin lesions in pure neuritic type of leprosy, there is greater chances of missing the diagnosis or misinterpretation of diagnosis as normal myalgia or arthralgia. Hence it is important for us to know the basis of diagnosis \& treatment of this condition.

\section{Methods}

\section{Case Report}

A 23 Year old male patient came with complaints of decreased sensation over the dorsum of right hand along with thinning \& weakness over the right hand for the past 10 years.

On elaborating the history, the patient had history of Multibacillary leprosy before 10 years, for which patient was advised 12 months of regular treatment with monthly follow up. But there was history of irregular treatment \& lack of proper follow up once there was subsidence of lesions.

Following the withdrawal of antileprotic medications, patient had developed gradual decrease in sensation over the dorsum of right hand along with weakness \& gradual thinning of the right hand over the past 10 years, which he initially did not take it too seriously.

Following increase in the symptoms, patient came for consultation.

Patient has no other associated comorbidities and there is no significant family history.

Sleep \& appetite regular. Bowel \& bladder habits normal.

On general examination, no obvious findings noted. Patient conscious, oriented, afebrile \& well built.

Systemic examination of cardiovascular, respiratory \& abdomen revealed no abnormalities.

\section{Neurological Examination}

Peripheral nerves get damaged more in leprosy, superficial nerves have more chance of getting damaged. Regions like the carpal tunnel, and the area in elbow where the ulnar nerve passes also have greater chances. When inflammation occurs, due to inflammation, there is compression and damage of nerve. On palpation, thickness and tenderness of nerve are important to be examined.

Palpation of Nerves: palpation for enlargement of nerves \& comparison of both sides should be done using pulps of the fingers.

The various nerve changes to look for include

- Increase in size

- Compression of nerve on palpation

- Single or both sides

- Nerve palpable measurement

- Regularity of nerves

- Tender on palpation

\section{Peripheral Nerves}

- Supraorbital

- Supratrochlear

- Great auricular

- Cervical

- Supraclavicular

- Median

- Ulnar

- Radial

- Sural

- Posterior tibial

- Common peroneal

- Lateral popliteal

Interpretation of findings: Ulnar nerve thickening was present over the right elbow on palpation.

Sensory testing: Semmes Weinstein monofilament were being used to measure light touch \& crude touch sensations.

There was diminished light touch sensation in the patient with awareness of crude touch.

Temperature Testing: Cold water of 5 degree Celsius \& hot water of 45 degree Celsius was taken in 2 test tubes \& tested for sensation. 
Both hot \& cold sensations were decreased over dorsum of right hand.

\section{Motor Examination}

- FACE-Paralysis of face muscles

Lion like facies

- UPPER LIMBS-Hand abnormalities like Claw hand

Wrist or finger drop

wasting of muscles

Digits shortened

- LOWER LIMBS-Gait abnormalities

Foot drop

Claw foot

Collapsing of arches

Shortened toes

Muscle wasting

Interpretation of Results: Deformity present over the right hand \& forearm since wasting of muscles present. No gait abnormalities noted.

Voluntary Muscle Testing: Testing for voluntary muscles is done for testing muscle power

Grade 3- movement possible without resistance was present over the Right forearm \& hand.

Grade 5 with normal power-was present over other areas.

\section{Dermatological Examination}

On dermatological examination, there was generalized xerosis with increased skin markings noted over the dorsum of right hand \& forearm with finely scaly, ichthyosis like skin changes.

There was mild pain $\&$ tenderness on palpation of ulnar nerve by flexing the elbow.

\section{Clinical Diagnosis}

After thorough clinical examination of the patient $\&$ confirming the absence of any obvious specific skin lesions of leprosy elsewhere, a clinical diagnosis of pure neuritic leprosy was made.

\section{Investigations}

Patient was advised biopsy. Punch biopsy was taken from the ulnar aspect of the dorsum of right hand of the patient.
High resolution ultrasound was done in both upper limbs.

Pt was advised complete blood count, liver function, renal function, Urine routine, HIV, VDRL or RPR tests.

\section{Results}

Histopathology findings revealed perivascular inflammatory infiltrate present in the dermis, with mild dermal fibrosis, occasional epineurial inflammation \& granuloma noted around the dermal nerve fibres. No acid fast bacilli were present in skin histopathology. Fite staining was done and revealed no lepra bacilli.

\section{Clinical Images of the Patient}
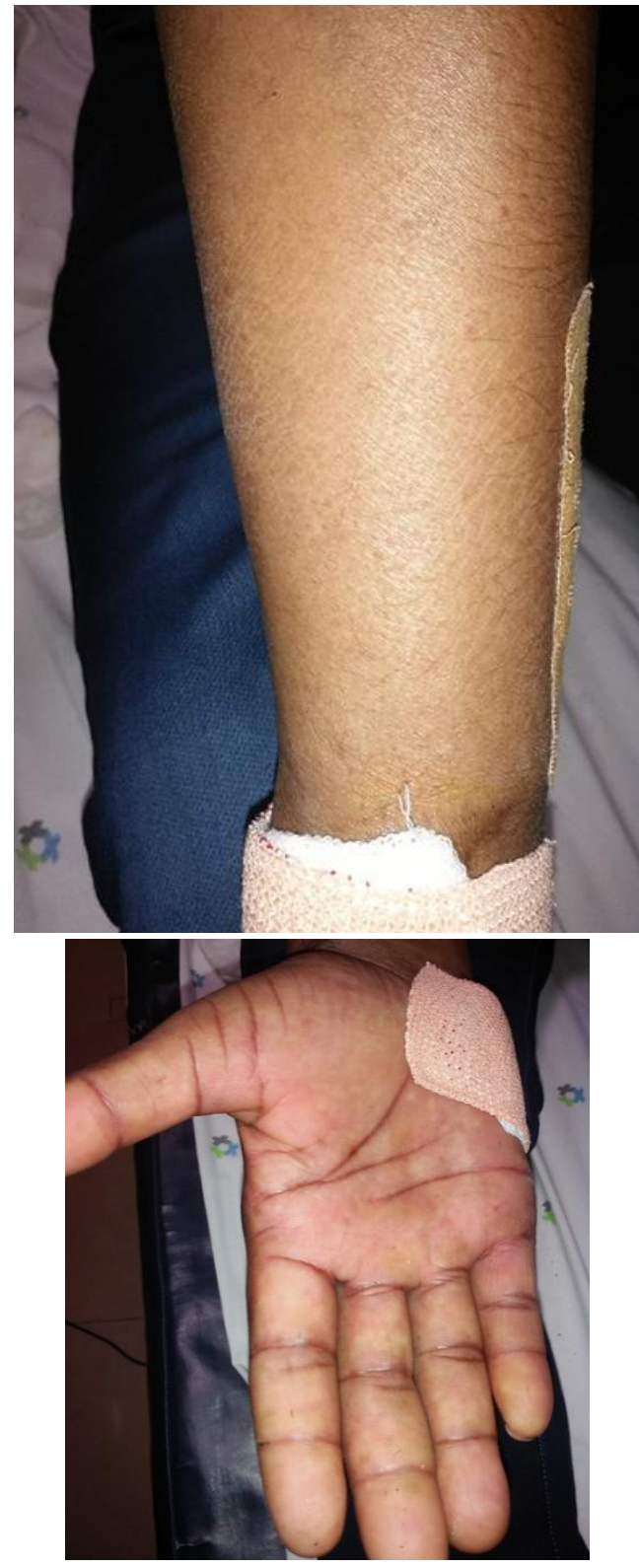


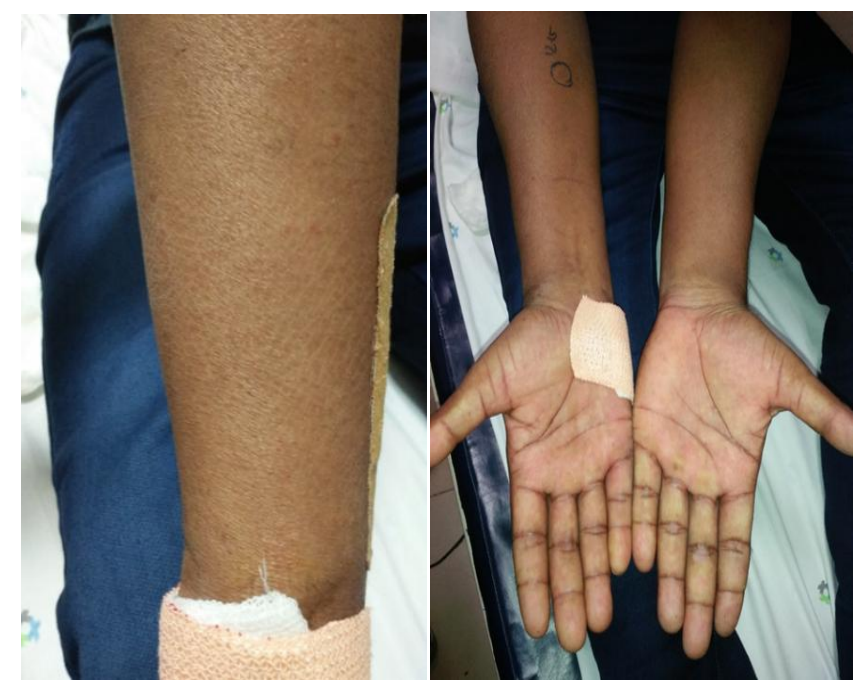

\section{Histopathology}
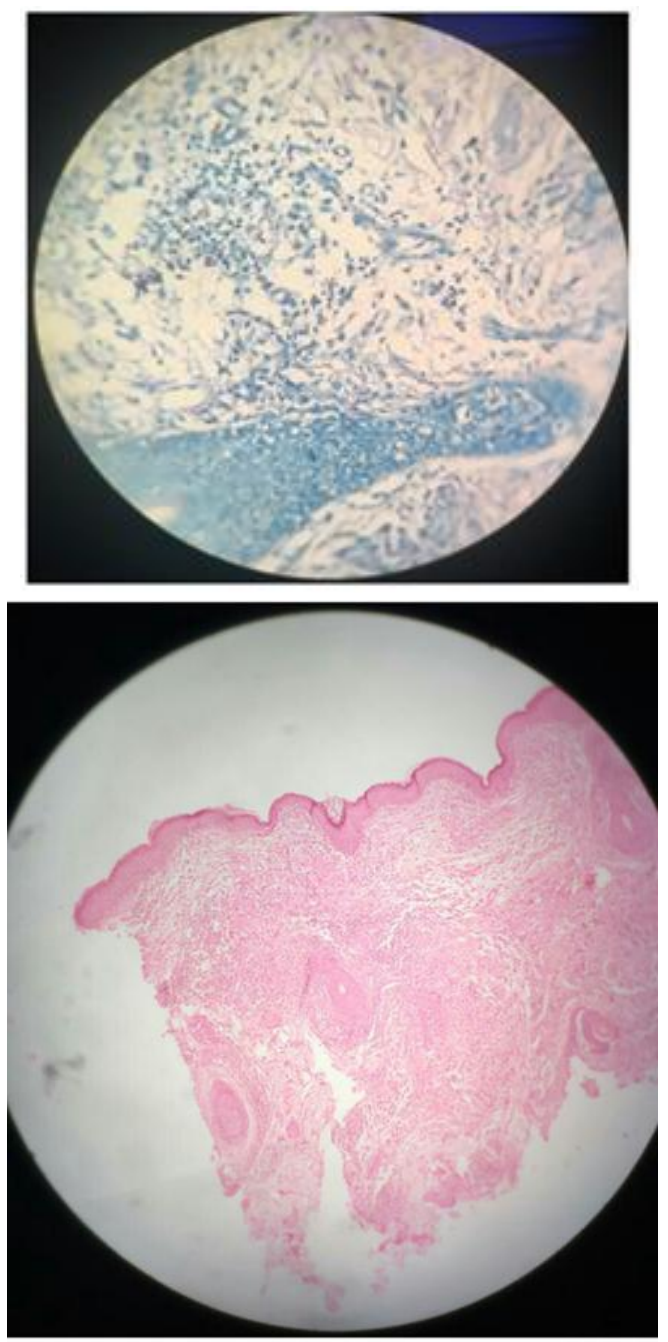

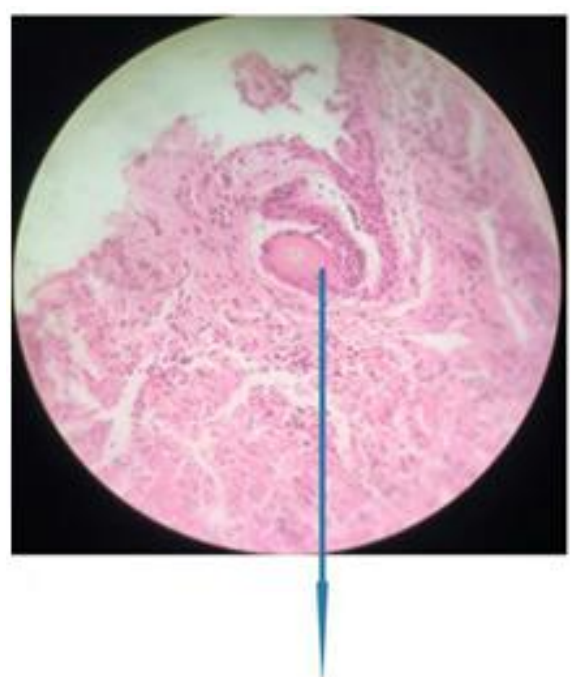

Granuloma around nerve bundle

High resolution ultrasound showed thickening of right \& left ulnar nerves with slowing present. Blood tests were normal \& revealed no abnormal values.

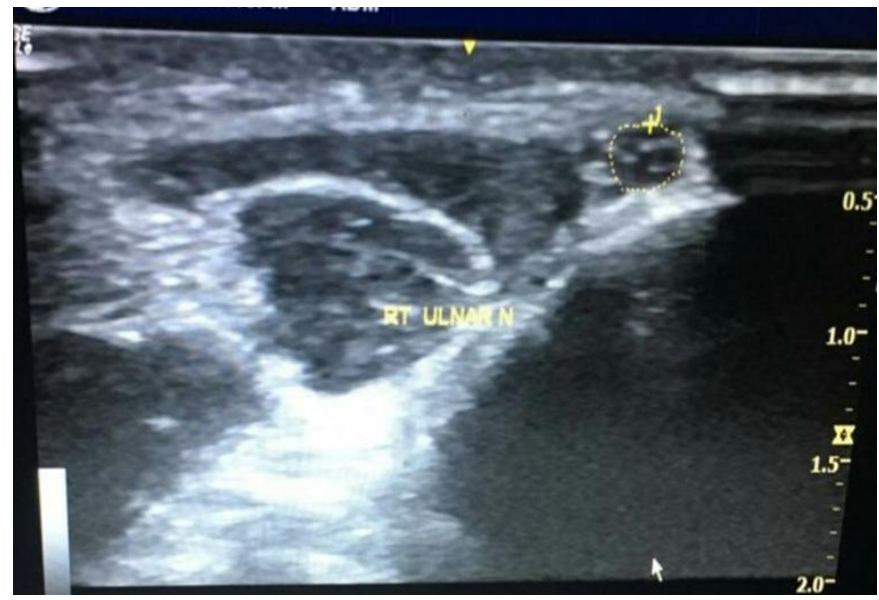

Thickened Right Ulnar Nerve

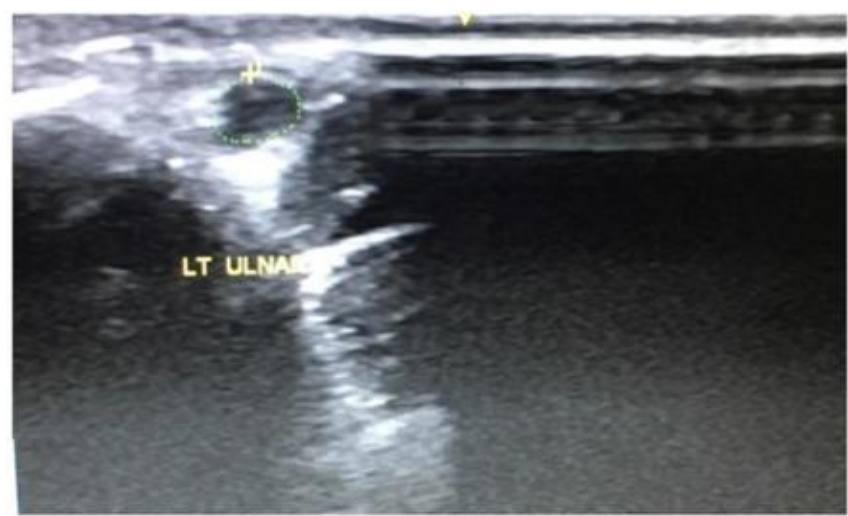

THICKENED LEFT ULNAR

NERVE 


\section{Treatment}

Considering the severity of the nerve affected, it is important to start Multibacillary regimen for leprosy at the earliest with regular treatment \& follow up for 12 consecutive months.

Prednisolone \& other oral steroids can also be given for 3-6 months to quickly reduce the inflammatory response present which will help in the recovery \& functioning of nerves affected in pure neuritic leprosy.

\section{Conclusion}

National Leprosy Eradication Programme played a vital role in the elimination of leprosy from India in 2005.But with the recent increase in the reporting of incidence of new cases \& patients with residual deformities due to irregular treatment \& relapse, it is a timely measure to come up with a strict $\&$ effective means to control leprosy, strictly adhere to treatment regimens in leprsosy as per protocol \& to reduce the deformities of patients suffering from leprosy by adequate treatment $\&$ rehabilitation measures.

\section{References}

1. Einar Wilder Smith. Diagnosis of pure neuriticleprosy. Neurol J Southeast Asia 2002;7:61-63

2. DEBAJYOTI CHATTERJEE*, GARGI KAPATIA*, et al. Histological spectrum of pure neuritic leprosywith atypical clinical presentation at a tertiarycare centre. Lepr Rev 2017 ;88;478-487.

3. Monalisa Hui, Megha S. Uppin, Sundaram Challa, Meena A. K.1, Subhash Kaul1 Pure neuritic leprosy: Resolving diagnostic issues in acid fast bacilli (AFB)-negative nerve biopsies: A single centre experience from South India.Ann Indian Acad Neurol.2015;18(3):292-297

4. Yogesh Tayade, N.V. Dravid, K.H. Suryawanshi, Sushil Mahajan, Rajeshwari K Pure Neuritic Leprosy: A Rare Case Report. International Journal of Scientific and Research Publications 2013; Volume 3(2):1-3. 